\title{
Género en lingüística Sistémico Funcional y en Sociorretórica: Apuntes para una didáctica de la lectura y la escritura en la universidad*
}

\author{
ILENE ROJAS GARCÍA** \\ zellene7@gmail.com
}

Recepción: 08 de febrero de 2016

Aprobación: 18 de marzo de 2016

Forma de citar este artículo: Rojas García, I. (2016). Género en Linguística Sistémico Funcional y en Sociorretórica: Apuntes para una didáctica de la lectura y la escritura en la universidad. Cuadernos de Lingüística Hispánica, (28), 93-114. doi: http://dx.doi. org/10.19053/0121053X.4910

* Artículo de reflexión.

** Licenciada en Español y Literatura, Magíster en Lingüística de la Universidad Tecnológica de Pereira. Doctoranda en Educación de la Universidad del Norte. Docente del departamento de Español de la Universidad del Norte; miembro fundador del grupo Estudios del Lenguaje y la Educación (Colciencias). 


\section{Resumen}

Si bien es evidente que las universidades colombianas están generando espacios de acompañamiento a los procesos de lectura y escritura de los estudiantes, es clara la necesidad de una reflexión teórica que permita sustentar las intervenciones en el aula. El presente documento explora los postulados de la Lingüística Sistémico Funcional y la Sociorretórica, como corrientes lingüísticas que han aportado al campo de la alfabetización académica en el entorno anglosajón y que son susceptibles de ser recontextualizadas para el ámbito nacional. Estudiar el concepto de género desde ambas corrientes, permitirá establecer categorías de análisis, así como herramientas para abordar el estudio de la realidad en la cual se inscriben los documentos. De esta manera, no solo se conseguirá un aprendizaje de los conceptos sino de los formatos discursivos y las condiciones que se establecen al interior de la interacción disciplinar.

Palabras clave: Alfabetización académica, Lingüística Sistémico Funcional, Sociorretórica, Género discursivo, Educación superior.

\section{Discursive gender in Systemic Functional Linguistics and Socio-Rhetoric: Notes for a Didactic Approach to Reading and Writing Skills at the University}

\section{Abstract}

While it is evident that Colombian universities are making an effort to create proper spaces to assess and enhance reading and writing processes in students, the need for a theoretical reflection that supports classroom interventions is clear. This document explores postulates from Systemic Functional Linguistics and Socio-Rhetoric, linguistic branches that have contributed to the academic literacy field in English speaking environments, but also offer possibilities of adaptation to the national context. Studying the concept of discursive gender from both perspectives will allow the establishment of categories and tools of analysis in order study the reality of the examined documents. In this way, not only the learning of the concepts, but also the learning of the discursive forms and conditions established in disciplinary interaction will be achieved.

Key words: Academic literacy, Systemic functional linguistics, Socio-rhetoric, Discursive gender, University education. 


\section{Genre en Linguistique Systémique Fonctionnelle et en Socio-rhétorique: Des notes pour une Didactique de la lecture et de l'écriture à l'université}

\section{Résumé}

Bien qu'il soit évident que les universités colombiennes sont en train de générer des espaces d'accompagnement aux processus de lecture et écriture des étudiants, il est clair que l'on a besoin d'une réflexion théorique qui permette de soutenir les interventions dans la salle de classe. Ce document explore les postulats de la Linguistique SystémiqueFonctionnelle et de la Socio-rhétorique, en tant que courants linguistique qui ont fait des apports au domaine de l'alphabétisation académique dans l'entourage anglo-saxon et qui sont susceptibles d'être reconstextualisés pour le territoire national. Étudier le concept de genre à partir de deux courants, permettra d'établir des catégories d'analyse, ainsi que des outils pour aborder la réalité dans laquelle les documents s'inscrivent. De cette manière, on réussira non seulement un apprentissage des concepts, mais aussi des formats discursifs et des conditions établies à l'intérieur de l'interaction disciplinaire.

Mots clés: Alphabétisation académique, Linguistique Systémique Fonctionnelle, Socio-rhétorique, Genre discursif, Éducation supérieure.

\section{Gênero em Linguística Sistêmico-Funcional e em Sócio-retórica: Apontamentos para uma Didática de leitura e escritura na universidade}

\section{Resumo}

Embora seja evidente que as universidades colombianas estão gerando espaços de acompanhamento aos processos de leitura e escritura dos estudantes, é clara a necessidade de uma reflexão teórica que permita sustentar as intervenções na sala de aula. 0 presente documento explora os postulados da Linguística Sistêmico-Funcional e da Sócio-retórica, como correntes linguísticas que tem aportado ao campo da alfabetização acadêmica no entorno anglo-saxão e que são susceptíveis de serem recontextualizadas para o âmbito nacional. Estudar o conceito de gênero desde ambas as correntes permitirá estabelecer categorias de análise, assim como ferramentas para abordar o estudo da realidade na qual se inscrevem os documentos. Desta maneira, não só se conseguirá uma aprendizagem dos conceitos, mas também dos formatos discursivos e das condições que se estabelecem ao interior da interação disciplinar.

Palavras chave: Alfabetização acadêmica, Linguística Sistémico-Funcional, Sócioretórica, Gênero discursivo, Educação superior. 


\section{Introducción}

Este trabajo se centra en la responsabilidad de la lingüística como ciencia que estudia el lenguaje, y que, junto con los procesos pedagógicos en las aulas universitarias, interesa al campo de la alfabetización académica. La preocupación inicial parte de las pocas revisiones existentes en el contexto colombiano acerca de los enfoques teóricos dirigidos a los procesos de alfabetización académica, desde la responsabilidad que atañe a la lingüística. En efecto, una revisión anterior (Rojas, 2015), puso en evidencia que las investigaciones sobre alfabetización académica en el país desconocen las corrientes teóricas del entorno anglosajón que, desde hace más de tres décadas, están avanzando en los estudios del discurso en el contexto académico y que ya se están recontextualizando en otros países de América Latina.

Frente a los procesos de alfabetización académica, es claro que la amplitud de áreas del saber sobrepasa las posibilidades de trabajo de los lingüistas, tanto por el desconocimiento de los contextos como de la dinámica académica propia de los diferentes ámbitos profesionales. Tampoco es posible delegar en los docentes de las disciplinas la responsabilidad en la formación de lectores y escritores, pues ellos no poseen un conocimiento especializado sobre la dinámica del lenguaje. Por tanto, hace falta un análisis profundo de los documentos que se estudian en la universidad. Tal análisis permitirá reconocer los aspectos discursivos y desde allí, sentar las bases para la intervención que trascienda el estudio descriptivo del texto e incluya los aspectos extratextuales que condicionan su sentido. Por tanto, interesa revisar el concepto de género discursivo desde dos enfoques teóricos que se ubican en el estudio del lenguaje con énfasis en los procesos pedagógicos para la lectura y la escritura en las disciplinas: la Linguística Sistémico Funcional (LSF), de la escuela australiana, y la Sociorretórica, en el campo norteamericano.

Como concepto que trasciende la gramática estructuralista, la noción de género es la piedra angular para la linguística contemporánea, más interesada en el papel semiótico del contexto comunicativo que en la explicación de la lengua como estructura normativa. Así, las relaciones que se tejen entre la realidad, la lengua, y su papel en el significado, son objeto de discusión en las distintas corrientes teóricas. Es necesario continuar las investigaciones en este campo, pues los conocimientos sobre género permiten potenciar los procesos de enseñanza y aprendizaje del lenguaje escrito, no como conocimiento abstracto, sino como mecanismo para actuar en sociedad. 


\section{La Lingüística Sistémico-Funcional (LSF)}

\subsection{Fundamentos epistemológicos de la Lingüística Sistémico Funcional}

Las huellas de la LSF se remontan a los inicios del siglo xx en los trabajos del antropólogo Malinowski y sus estudios sobre el contexto de cultura y el contexto de situación. Malinowski (1923) sugirió que el contexto debía ser estudiado en dos planos: el inmediato de la acción comunicativa, y el cultural donde se desarrolla la actividad social mediada por el lenguaje. Ambos planos dan cuenta de los aspectos externos al texto (sociales, culturales, políticos, históricos, económicos, psíquicos, etc.) que lo impregnan de sentido. Posteriormente, el lingüista Firth, retomó estos planos del contexto y reelaboró el concepto, adecuándolo y precisándolo para las ciencias del lenguaje. Así, definió el contexto de situación como una serie de factores externos al texto que condicionan el uso de la lengua, entre ellos propone: los participantes, la acción verbal y no verbal, los objetos relevantes y los efectos de la comunicación (Ghio y Fernández, 2008, p. 34). En este sentido, para Halliday (1982), el contexto de situación se encuentra constituido por "aquellas propiedades generales de la situación que funcionan colectivamente como determinantes del texto, en cuanto especifican las configuraciones semánticas que el hablante conformará típicamente en contextos del tipo dado" (p. 145).

Por otro lado, la LSF está influenciada por los análisis del control simbólico adelantados por Bernstein (2000). Para el sociólogo, la ideología es entendida en términos de las relaciones que se tejen al interior de los contextos y en los contextos entre sí; relaciones que penetran todos los niveles del significado. Así por ejemplo, en el ámbito cotidiano, el poder puede estar condicionado por la edad, el género u otras marcas de estatus. Pero en un campo más amplio, las relaciones de poder están mediadas por instituciones como la ciencia, la industria o la administración, de modo que el poder pasa a depender del nivel educativo; sin embargo, el acceso a la educación, a su vez, se encuentra condicionado por la posición del individuo en relación con el poder socioeconómico. Por tanto, el ejercicio del poder está condicionado por el lugar en las jerarquías sociales y la prominencia de la voz en la vida pública. En situaciones específicas, el propósito del lenguaje transita entre dominar o conceder autoridad y demandar o prestar atención a otros. Así, la ideología corre a través del conjunto entero del lenguaje en el contexto social, diferenciando los sujetos sociales en jerarquías de poder, control, estatus, autoridad y prominencia (Gee y Handford, 2012, p. 210). En este sentido, la LSF tiene como objetivo intervenir en la redistribución de los recursos semióticos a través de la educación, más que solo criticar los recursos de poder. 
Por tanto, la LSF incluye el contexto social e ideológico para explicar los aspectos linguísticos en relación con la enseñanza de la lengua. Desde esta perspectiva, se trata de una lingüística funcional en tanto se centra en el uso que hacen los hablantes, y sistémica puesto que revisa la forma como estructuran la lengua según las condiciones del contexto. En consecuencia, se resalta la concepción dialéctica del lenguaje; por un lado, el contexto social se materializa a través del texto, activando una serie de elecciones que el hablante debe realizar para cumplir su propósito comunicativo; y a la inversa, el texto es una realización denotativa del contexto social, de modo que las emisiones lingüísticas van a afectar el entorno de la interacción (Halliday y Hassan, 1985, p. 47).

Cabe señalar, sin embargo, que para la LSF, el concepto de función es interno al lenguaje como propiedad, manteniendo un marcado interés en los aspectos estrictamente lingüísticos. Halliday (1989) sostiene que la función es interpretada como propiedad fundamental del lenguaje en sí mismo, un componente de base para la evolución del sistema linguístico. En la misma línea, afirma que la gramática es la unidad central de procesamiento del lenguaje, la central eléctrica donde los significados son creados; es natural que el sistema de sonidos y escritura a través del cual se expresan los significados, reflejan la estructura de la gramática (Halliday, 2014). De allí que su foco de estudio es el lenguaje proyectado a la vida social.

\subsection{El género en la LSF}

Esta perspectiva, sistémica y funcional, que se relaciona directamente con el contexto y con las relaciones de poder, se concibe a manera de capas superpuestas desde donde la realidad más exterior que es el contexto de cultura, se va configurando paulatinamente a partir de una serie de condiciones hasta llegar a la emisión lingüística del hablante. Tales capas son denominadas por Halliday como estratos o niveles del lenguaje. La primera conexión entre la actividad humana y el lenguaje es aquella que permite relacionar el contexto de cultura con el texto, esto es, el género. Para Martin y Rose (2012) el género es definido como un

proceso social, orientado a objetivos y realizado en etapas. Social porque inevitablemente estamos tratando de comunicarnos con otros, orientado a objetivos porque siempre tenemos un propósito al escribir y nos sentimos frustrados si no lo cumplimos, y realizado en etapas en tanto que usualmente nos toma más de un paso alcanzar nuestros objetivos. (p. 54).

Se trata entonces de un estrato superior que contiene el aparato linguístico y su funcionalidad, donde la cultura y el contexto de comunicación son reconstruidos en un estrato socio-semiótico. 
Continúa la conexión entre el contexto de situación que se materializa en el registro, se trata del estrato donde aparecen las dimensiones de campo, tenor y modo y su correspondiente metafunción. El campo, que conforma la metafunción ideacional, da cuenta de los hechos sucedidos a nivel de: la naturaleza de la actividad social y semiótica, así como el dominio de la experiencia con la cual se relaciona la actividad; se evidencia en el texto a partir de las ideas, conceptos y definiciones que se plantean. El tenor, a nivel de la función interpersonal, se refiere al rol que desempeña quienes hacen parte de la situación, las relaciones sociales que se pueden sobrentender del texto y las intenciones sociales; también son relevantes los valores que los actantes incluyen en la interacción (ya sea neutro o cargado, positiva o negativamente). El modo, determinado por la función textual, incluye la estructuración del texto, según: si constituye o apoya la actividad social, los usos retóricos del lenguaje, el turno conversacional, la coherencia entre las cláusulas, así como el medio y el canal por el cual se transmite el mensaje (Halliday, 2014). Así pues, el análisis del registro da cuenta de la forma como prácticas sociales concretas obligan a usar recursos lingüísticos determinados.

Por último, la experiencia del contexto cultural y de situación, se convierten en lenguaje al atravesar el estrato de la semántica; posteriormente, el significado es convertido en palabras en el estrato de la lexicogramática; finalmente, la fonética es la base de la estratificación (Halliday, 2014). El siguiente diagrama esquematiza las relaciones establecidas por la LSF:

Figura 1. Género, registro y lenguaje.

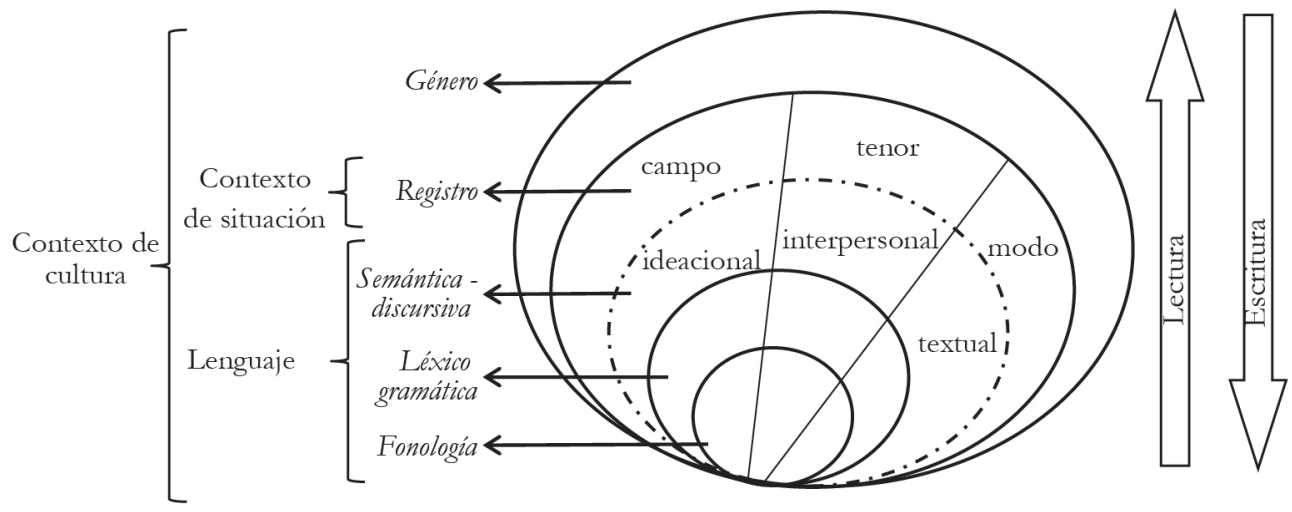

Adaptado de Martin y Rose, 2003, p. 309 y Moyano, 2014.

Cabe resaltar que, la división en estratos no implica en absoluto el estudio de sus componentes por separado, sino la posibilidad de analizar con mayor detenimiento todos los factores involucrados en la emisión linguística. De esta forma, se facilita el estudio 
acerca de cómo interactúan entre sí y cómo afectan, tanto el sistema de la lengua como el de sus significados profundos en el marco de la lengua en uso.

La interpretación de esta secuencia interrelacionada de estratos puede ser transferida al campo de la alfabetización académica, analizando estos aspectos en doble vía: hacia arriba, al momento de enfrentarse a una lectura en la que se parte del material grafológico para inferir de allí los procesos sociales que lo configuran; y hacia abajo, en tanto se parte de un requerimiento social para producir un texto.

\subsection{El género de la LSF en clave didáctica}

A partir de esta conceptualización relacional del sistema de la lengua, la Escuela de Sidney, derivada de los trabajos de Halliday, ha enfocado sus estudios en el desarrollo de programas de intervención en el aula, por lo que se denomina Pedagogía de Género. Desde allí se afirma que reconocer los aspectos de la lengua a nivel genérico, facilita y optimiza su transferencia y aplicación en entornos educacionales, en tanto permite trabajar desde la consciencia funcional de las elecciones lingüísticas.

Los trabajos de esta corriente han aportado descripciones detalladas de algunos géneros usados al interior de la educación básica, en las que se ponen en evidencia las etapas de la estructura del lenguaje, sus funciones y las realizaciones lingüísticas, directamente vinculadas con las dimensiones de campo, tenor y modo. Martin y Rose (2003) resaltan el carácter social del género, al definirlo como "los diferentes tipos de texto que difunden distintos tipos de contextos sociales" (p. 8). Estos géneros son aprendidos en la interacción y sus particularidades se constituyen como modelos de uso que pueden variar desde las conversaciones cotidianas, hasta las esferas del lenguaje científico o político. Esta descripción de los géneros se realiza a partir de la relación que se establece entre ellos, pues las diferencias ayudan a definir de qué género se trata (Gee y Handford, 2012).

En el contexto escolar, Martin y Rose (2008) han descrito básicamente cuatro tipos de género, de acuerdo con su propósito social y con su objetivo para la enseñanza de la lectura y la escritura. El primero es el género de los relatos, cuyo objetivo central es el entretenimiento, por lo que se analizan principalmente los recursos lingüísticos orientados a este objetivo. A nivel estructural, el relato puede iniciar opcionalmente con una etapa de orientación donde se presenta una secuencia de actividades que van a suceder, pero varía en la forma como se interrumpe y cómo se responde a esta interrupción. Esta secuencia generalizada, cuenta con una serie de variaciones que conforman el género de la narración. Entre ellas se encuentran los recuentos de experiencias personales, anécdotas, ejemplos y observaciones; estos textos no resuelven el conflicto, por lo que se reserva el término "narrativa" únicamente para el modelo genérico que sí lo hace. 
Un segundo grupo de géneros es el informativo, o aquellos que tienen la función de proveer información, sea esta de carácter histórico, explicativo o documental, especialmente relevante en el contexto académico. Dentro de este género se encuentran los reportes, orientados a clasificar y describir la realidad; las explicaciones, acerca de cómo suceden los hechos a nivel de causas y efectos; los procedimientos, que van desde los instructivos acerca del manejo de aparatos, hasta la legislación y los planes desarrollados en contextos comerciales o administrativos.

Al tercer grupo le interesa establecer los procedimientos requeridos para llevar a cabo determinadas actividades. Entre ellos están los géneros orientados a proponer cómo realizar una actividad, y aquellos que describen la forma como se llevó a cabo. Las explicaciones, reportes y procedimientos en ciencia y tecnología incluyen diagramas, cuadros, fotografías o mapas que soportan la interpretación verbal. Y al mismo tiempo, los soportes visuales siempre están acompañados de un texto verbal que los interpreta. De allí que Martin y Rose (2008) los describan desde tres perspectivas: aquellos construidos por imágenes visuales, aquellos que caracterizan textualmente imágenes visuales y aquellos que establecen una relación entre los géneros verbales y visuales en textos multimodales.

Finalmente, se presentan los géneros cuya función es evaluar los discursos, ya sea, validar las respuestas para los textos de tipo expositivo, o valorar las opiniones, en el caso de la argumentación. En estos géneros se negocia la posición en el discurso público. Mientras la exposición está organizada alrededor de las ideas presentadas sobre un mismo punto de vista, la argumentación se sustenta alrededor de posiciones en conflicto. En el marco de esta dinámica, se incluye una serie de valoraciones que refuerzan la posición del autor y controvierten la de sus opositores. Esta es una fuerte herramienta en el discurso ideológico, pero también juega un papel esencial al interior del sistema educativo, tanto para los investigadores, como mecanismo para promocionar su trabajo, como para los estudiantes que buscan demostrar su conocimiento (Gee y Hanford, 2012). Es claro que un mismo texto puede incluir múltiples propósitos, pero el género hace referencia al objetivo principal bajo el cual los demás estarían actuando como soporte. Los géneros descritos por la Escuela de Sidney son diversos, pero constituyen solo una fracción del repertorio de géneros disponibles para los miembros de una cultura.

Durante las últimas décadas, la Pedagogía de Género se ha enfocado en diseñar aspectos metodológicos que permitan a los estudiantes comprender el contexto cultural, las etapas y las herramientas del lenguaje de los textos estudiados. De allí que se han generado modelos como el de Martin y Rose (2012) en el marco del proyecto Reading to Learn. Los autores presentan la secuencia didáctica como un ciclo guiado por el maestro. El segmento inicial es la selección del género que va a ser abordado y el campo en el que se 
inscribe. Luego se deconstruye a partir del análisis de las relaciones entre las dimensiones constitutivas del discurso, como mecanismo de acción en el contexto cultural. Finalizada la etapa de lectura analítica, se prosigue con la mediación para la escritura, para esta tarea se conserva el género elegido. El ejercicio se realiza a través de la ejercitación en conjunto, modelado por el docente, que dará paso al trabajo independiente de los estudiantes; aquí, el estudiante puede tomar como modelo las características genéricas y las estrategias de escritura usadas por el maestro en la construcción conjunta. Para terminar, se propone generar espacios de discusión orientados a valorar los textos a nivel de sus usos reales y la reflexión sobre componentes ideológicos. Para ello se toma como modelo metodológico el ciclo de enseñanza y aprendizaje del proyecto de Lenguaje y Poder Social de Martin y Rose (2012).

Figura 2. Ciclo de enseñanza y aprendizaje del proyecto de Lenguaje y Poder Social.

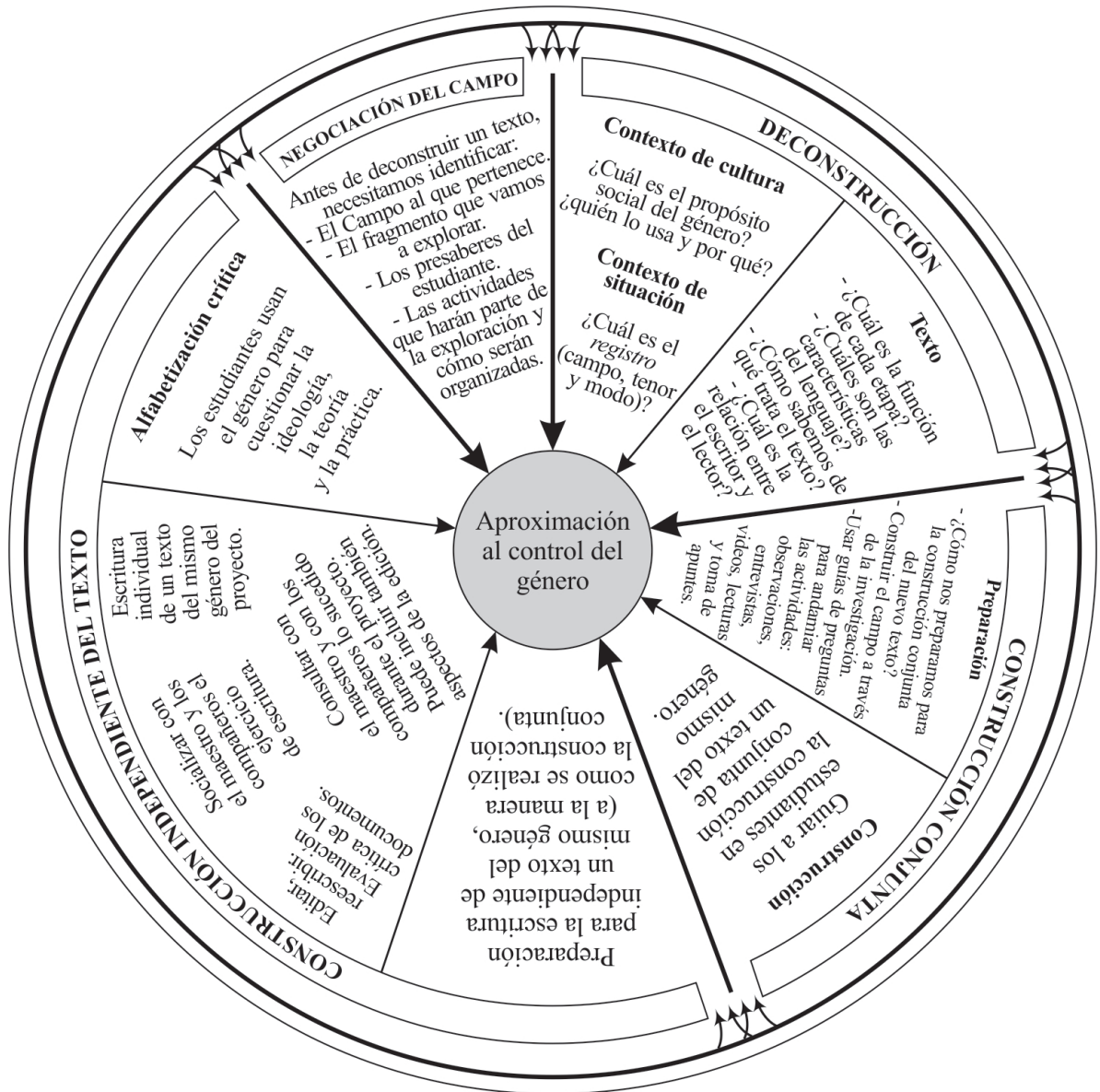

Tomado de Martin y Rose (2012, p. 65) 
Como se ve, en el ámbito educativo, la Pedagogía de Género ha contribuido en la descripción de textos que cumplen con objetivos didácticos (aún de manera indirecta). Tales estudios permiten direccionar la enseñanza de la lengua hacia objetivos reales de comunicación, desarrollo del pensamiento y uso del lenguaje. Sin embargo, es clara la centralidad en el texto como unidad de análisis en la que se ponen de manifiesto la cultura y la ideología; a nivel de la alfabetización académica, quizá esto no sea suficiente, hace falta estudiar desde afuera el contexto académico en el que se desarrollan los textos disciplinares, pues se trata de un ámbito casi totalmente desconocido para los estudiosos del lenguaje, y que no es posible determinar únicamente desde el análisis textual.

\section{La Sociorretórica}

\subsection{Fundamentos epistemológicos de la Sociorretórica}

La sociorretórica reflexiona sobre la naturaleza de los seres humanos como organismos activos, comunicativos y simbólicos. Desde allí, sostiene que el discurso se genera al interior de los sistemas sociales, en la interacción; pero también, el discurso en sí mismo genera acciones sociales, al producir textos (Bazerman, 2012a, p. 123). Esto quiere decir que el lenguaje, simultáneamente, es construido y construye la vida social.

El concepto del lenguaje como acción tiene su base en la teoría de los actos de habla de Austin (1962) y Searle (1969), el primero, describe cómo las palabras llevan a cabo las acciones; el segundo identifica y delimita los actos de habla. Los usuarios del lenguaje (con capacidades neuropsicológicas e historias individuales de la experiencia linguística) en el curso de la interacción, evocan los recursos del lenguaje que están social y culturalmente disponibles y que han sido tipificados a través de años de circulación social; al mismo tiempo, los individuos construyen significados a partir de la percepción de nuevas situaciones y de su participación activa en ellas. Por tanto, el significado es una negociación entre la distribución pública de los recursos linguísticos y las prácticas del lenguaje ocurridas en el contexto específico, y en dependencia recíproca con prácticas individuales.

En este sentido, el estudio del discurso considera a la gente en la interacción, buscando: la forma como construyen significado para lograr sus fines, los mecanismos para establecer conexiones con el otro orientados a conseguir el entendimiento, y, el proceso por el cual se lleva a cabo esta construcción. La coordinación de tales factores determina la complejidad del mundo discursivo como lo conocemos. Sobresale entonces un interés por el mundo social que encuentra apoyo en los estudios de Rorty (1989). Para el autor, el conocimiento es un constructo social que responde a necesidades, objetivos y contextos comunales. En la misma línea, su discípulo Bruffee (1986) señala que los 
conceptos, ideas y teorías sobre la realidad del mundo son construcciones del lenguaje generados por comunidades de conocimiento, utilizados para mantener la coherencia de la comunidad. Paralelamente, la sociorretórica incluye las afirmaciones clásicas sobre los hechos sociales a partir de las teorías de Durkheim (1986).

Siendo el lenguaje una actividad de cohesión social, se hace indispensable reflexionar en torno a la transferencia del sentido del hablante que es susceptible de múltiples interpretaciones por parte del interlocutor. Por ello, se retoman los estudios de Schütz (1932), quien plantea que las acciones realizadas por el sujeto contienen un significado subjetivo que consiste en una autointerpretación de la vivencia. Por este carácter subjetivo, el significado al que el actor apunta con su acción es distinto al significado que otros le dan, y para ser interpretado, se requiere comprender las vivencias del otro a partir de las propias; pero también, es necesario utilizar las indicaciones exteriores que permitan reconocer sus experiencias. Así, Shütz (1932) declara: "Solo aprehendo las vivencias del otro mediante la representación significativo-simbólica, y considero su cuerpo o cualquier artefacto cultural que él haya producido como un campo de exposición de esas vivencias" (p. 130). Por tanto, cualquiera sea el contexto de significado interior del cual se genera la acción comunicativa, tal contexto adquiere su validez en la medida en que se relaciona con el significado correspondiente que está en la mente de la otra persona. Claramente, este último contexto debe ser el mismo dentro del cual se construyen las actuales vivencias del otro (Shütz, 1932). El enunciado se considera como exitoso cuando las partes deciden que sus necesidades o acciones se cumplen satisfactoriamente, o cuando el grado de aproximación logra los propósitos prácticos (Schutz y Luckman, 1973).

En el mismo sentido, se tienen en cuenta los postulados de Vygotsky $(1962,1978)$ particularmente en relación con su interés en la historia de las formas culturales. Los estudios vygotskyanos sobre la cooperación linguística son esenciales, para generar un modelo acerca del uso científico de la lengua. A partir de allí, Bazerman (1988) sostiene que la dinámica discursiva se logra a través del despliegue de actividades sociales con personas coordinadas tanto cognitiva como conductualmente; sin embargo, para que la cooperación tenga éxito, no es suficiente con compartir el significado de las palabras y las operaciones sintácticas, se requiere además revisar cómo se aplican esas palabras generalizadas en situaciones específicas y cómo deben realizarse en la acción, en función de las rutinas que organizan la actividad. En relación con los postulados de Shütz, es necesario poder ver lo que el otro ve, para orientar patrones y características específicas. Se requieren entonces conocimientos y experiencias compartidas que faciliten la creación del esquema perceptual. 
Paralelo a la concepción del lenguaje como mecanismo de cohesión social, la sociorretórica analiza el hecho lingüístico en su desarrollo histórico. Esto es así porque las personas usan los textos para crear nuevas realidades de significación, relación y conocimiento, de modo que, en la medida en que se modifican dichas realidades, se transforman también los textos. De allí que resalte las prácticas regularizadas de grupos de usuarios de la lengua, como directamente dependientes del tiempo y el espacio. Se trata de formaciones transitorias, en permanente evolución, endémicas de las instancias locales y usadas creativamente con un objetivo específico según cada usuario, en una serie específica de circunstancias.

\subsection{El género en la Sociorretórica}

El concepto de género desde la sociorretórica, parte de la reflexión de Bazerman (2012b) en torno a la invisibilidad que tiene el escritor frente a su interlocutor. Al momento de la lectura, el autor se presenta solo como un acto de imaginación basado en pistas limitadas ofrecidas tanto por el texto en sí mismo, como por el conocimiento previo del lector. Las pistas comunicativas para la alineación exitosa entre las intenciones del autor, el texto y la comprensión del lector, solo pueden realizarse a través de la disposición de las letras en palabras, frases y unidades dispuestas en el texto, así como los mecanismos de puntuación y la disposición gráfica. Sin respuesta interactiva inmediata, no es posible reparar, modificar o ampliar el significado para optimizar la comprensión. La insuficiencia de los signos lingüísticos y la distancia con el receptor, a menudo dejan al escritor sin saber si el documento producido evoca los significados y efectos deseados; desde la recepción, el lector lucha con la interpretación de lo que habría sido pretendido por el autor. Esta distancia se incrementa cuando el texto es complejo o se encuentra en un lenguaje poco conocido por el lector.

A pesar de estas limitaciones del texto escrito para ser interpretado solo de forma aproximada por personas diferentes en lugares apartados y con intencionalidades dispares, ha demostrado ser sólido para permitir la comunicación, incluso, de pensamientos tan abstractos como la filosofía, las teorías científicas, la planificación y coordinación de proyectos o el mantenimiento de instituciones (Bazerman, 2012b). Esta falta de correspondencia entre los límites del lenguaje y sus posibilidades, desemboca en el problema que enfrenta el lector para develar el sentido implícito del texto, más allá de las palabras escritas. Desde el enfoque sociorretórico, se trata de una cuestión de reconocimiento de situaciones y acciones dentro de las cuales se movilizan los significados a través de los signos. En este sentido, el interés de la sociorretórica se encuentra en la concepción del lenguaje como: hecho social, forma de acción y proceso dinámico en constante movimiento. 
Bazerman (2012a) señala que se suele identificar los géneros por medio de ciertas características especiales indicadoras del tipo de mensaje que se puede esperar; sin embargo, entender los géneros tan solo desde un número fijo de características, produce una visión de ellos como elementos "atemporales e iguales para todos los receptores" (p. 132): el saber de la comunidad cambia con el tiempo, así como los géneros y las situaciones, también el conocimiento común varía en cada persona o incluso en la misma persona bajo circunstancias distintas. Por tanto, la definición de género como un grupo de características textuales, distancia el papel de los individuos cuando hacen uso del lenguaje y crean significado, "ignora las diferencias de percepción y comprensión, el uso creativo de la comunicación para alcanzar necesidades nuevas percibidas en circunstancias también nuevas e ignora el cambio de comprensión de los géneros con el tiempo" (Bazerman, 2012a, p. 132).

Cabe resaltar que, la sociorretórica no abandona completamente las primeras concepciones de la linguística acerca de los géneros como tipos o clases de discurso, similares en forma y contenido; sin embargo, no tiene un interés especialmente taxonómico, pues la individualidad de los factores presentes en la construcción de un texto, los hace difícilmente homogenizables. La validez de la teoría sociorretórica en el estudio del género es su énfasis en los aspectos sociales e históricos de la retórica; de tal manera que la descripción del género no se centra en la sustancia o la forma del discurso sino en su vinculación con un tipo de actividad. Por ello, examina la relación entre el género y la situación recurrente; desde allí, el género se define como acción retóricamente tipificada, capaz de reconocer y conectar las regularidades en los tipos de discurso (Miller, 1984). Lo anterior permite un entendimiento social y cultural más amplio de la lengua en uso (Freedman y Medway, 2005), es decir, se hace posible desempaquetar los complejos factores sociales, culturales, institucionales y disciplinares que están en juego durante la producción de determinados tipos de escritura.

Por su parte, Miller (Bazerman y Miller, 2011) sostiene que esta definición permite "reflexionar sobre el modo como uno realiza una acción y responde a ella [...] tanto la producción como la recepción son importantes para poder pensar el género como acción" (p. 16). A renglón seguido, Bazerman advierte que, al trasladar el estudio del género del texto hacia la percepción del creador y del receptor, el género se transforma en una categoría de reconocimiento psicológico. Además, al tratarse de prácticas sociales que requieren un cierto nivel de alineación entre las personas a lo largo del tiempo, es necesario comprenderlos como categoría de reconocimiento social, de acuerdo social. Finalmente, Bazerman sostiene que "los enunciados son delimitados, tienen comienzo y fin, ocupan un lugar definido en el tiempo y en el espacio y se los percibe como portadores de algún 
sentido”. En consecuencia, los géneros son: 1. Categorías de reconocimiento psicosocial; y 2. Categorías de enunciados, que hacen parte de actividades socialmente organizadas (Bazerman y Miller, 2011, p. 18). Tales categorías son útiles para definir patrones extendidos funcionales en los distintos sistemas sociales que configurarán el género.

Además de este concepto, Bazerman (2012a) propone una serie de elaboraciones superpuestas que permiten abordar también aspectos particulares. El primero es el grupo de géneros, entendido como una colección de textos que se producen desde un rol particular; el segundo es el sistema de géneros, compuesto por varios grupos de géneros usados de manera conjunta y que se relacionan a través de esquemas de producción, flujo y uso de estos documentos; finalmente se define el sistema de actividad, es decir, el marco de trabajo en que se producen los textos; mientras más alto el nivel educativo y más profundo el conocimiento profesional, mayor relevancia tendrán los géneros escritos. Cada texto comprendido de manera exitosa crea un hecho social, es decir, acciones sociales significativas que se logran a través del lenguaje. Cabe resaltar que, para lograr el éxito en la comunicación, se requiere una alineación entre los actos locutivos, ilocutivos y perlocutivos según la teoría de la Austin y Searle, mencionada en el apartado anterior; si existe algún desfase entre estos factores, no podrá existir el hecho social compartido.

Para garantizar la coordinación de los actos de habla, el recurso utilizado es actuar de maneras típicas, es decir, "patrones comunicativos con los que otras personas están familiarizadas" (Bazerman, 2012a, p. 131), de modo que sea factible reconocer con mayor facilidad el mensaje que se quiere transmitir y el objetivo comunicativo que se busca lograr. Estos patrones se refuerzan mutuamente y son los que van a constituirse como géneros.

Al crear formas tipificadas, o géneros, también tipificamos las situaciones en las que nos desenvolvemos [...] A este proceso de movilización hacia formas estandarizadas de expresiones que son reconocidas como las que llevan a cabo ciertas acciones en ciertas circunstancias y hacia entendimientos estandarizados de las situaciones se le llama tipificación (p. 131).

Claramente, el análisis del género parte de las similitudes de forma, pero el concepto de tipificación induce a realizar un estudio más profundo: revisar las similitudes de contenido o sustancia y las similitudes de acción (Bazerman y Miller, 2011). Entonces, la estabilidad comunicativa no es proporcionada directamente por el género, sino por el sistema de actividad de la que el género hace parte, un conjunto de recursos sociales necesarios para que la gente pueda entenderse entre sí, coordinar actividades y compartir significados orientados a propósitos prácticos (Bazerman, 2012a). 


\subsection{El género de la Sociorretórica en clave didáctica}

A partir de esta teorización acerca del género como acción retóricamente tipificada, los estudios sociorretóricos se convierten en un esfuerzo internacional por avanzar en la investigación sobre las prácticas de lectura y escritura en la universidad, al interior del movimiento WAC (Writing Across the Curriculum). En los trabajos inscritos en esta línea, se advierte un interés por la enseñanza de los géneros escritos en el campo de las ciencias sociales y naturales, no como técnicas para ser copiadas, sino como indicios de las formas de conocimiento, la epistemología y las acciones sociales propias de cada disciplina. El énfasis se presenta en las dimensiones sociales de las disciplinas: cómo y por qué se escribe en las profesiones (Russell y otros, 2009).

Como su nombre lo indica, el movimiento WAC tiene su principal interés en los procesos de escritura de los textos que deben producir tanto los estudiantes universitarios como los profesionales al interior de cada campo del saber. En este sentido, se han desarrollado experiencias de trabajo en el aula desde la educación primaria hasta los estudios superiores, prestando especial atención a los documentos del campo de la ciencia y con un interés marcado en concebir la escritura como un mecanismo de acceso al conocimiento. Además, se han adelantado análisis y propuestas didácticas relacionadas con los textos propios del campo de la salud, las matemáticas, las ciencias económicas y la historia (Bazerman y otros, 2005; Navarro, 2014). Paralelamente, trabajos alrededor de los contextos sociales de participación democrática como mecanismo para reflexionar sobre asuntos de interés general; así como los procesos de escritura en el campo profesional y laboral (Bazerman y otros, 2012).

Frente a estos aspectos, Bazerman (1988) encuentra que las prácticas de alta especialización de la escritura académica surgieron dentro del complejo de la evolución de la actividad científica, y esos mismos principios de significado situado determinan las decisiones en la dinámica del aula universitaria. En este sentido, las prácticas del lenguaje evocan los mundos de conocimiento a los que pertenecen y es precisamente allí donde se hacen indispensables los estudios de género, pues no se tiene un conocimiento suficiente sobre los géneros y las actividades de los campos que son importantes para los estudiantes (Bazerman 2012a). La opción es incrementar este conocimiento a través de la investigación, examinando más textos de una forma regularizada, entrevistar y observar a más escritores y lectores y documentar etnográficamente la manera en que los textos son utilizados en las distintas institucionalidades (Bazerman 2012).

Ayudar a los estudiantes a leer y escribir en las situaciones con las que están menos familiarizados (como los de las disciplinas y las profesiones) requiere hacer explícitos los 
sistemas de actividad en donde estas se producen. Al respecto, Miller (Bazerman y Miller, 2011) sostiene que, si el género se concibe como un hecho social, entonces es necesario situar la enseñanza de la escritura también como un hecho social, de modo que el estudiante encuentre el sentido de la actividad y se esfuerce para llevarlo a cabo. Este reconocimiento desemboca en una necesaria consciencia de la interacción entre el docente y los estudiantes. Así, las acciones del docente actúan como modelo para los estudiantes, en sentido inverso, la respuesta de los estudiantes a las tareas asignadas, servirá de base para las siguientes acciones. Además, el profesor puede presentar los objetivos de leer y escribir determinado género, así como las posibilidades de recepción por parte de los lectores. Miller (Bazerman y Miller, 2011) también propone tener en cuenta los géneros que el estudiante ya conoce para hacerle consciente de las tipificaciones sociales propias del mundo, posteriormente considerar las tareas curriculares o los objetivos de aprendizaje que requiere el estudiante, junto con los géneros que deben aparecer en el siguiente nivel. El papel del profesor, entonces, es gestionar el sistema de actividades en el aula de clase, y en esta medida pueda crear situaciones lo más cercanas posible a las prácticas reales, con géneros significativos que los promuevan como hablantes, escritores, ingenieros, abogados, médicos, etc.

Comprender los géneros y la forma como trabajan en los sistemas y circunstancias para las que fueron diseñados, permite al estudiante en tareas de escritura: 1. Cubrir las necesidades de la situación de manera que el texto pueda ser comprendido por otros; 2. Entender cuándo los textos, aparentemente bien escritos, no funcionan; 3. Diagnosticar los sistemas de actividades para determinar si un grupo específico de textos son redundantes o confusos, si se requiere eliminar, adicionar o modificar detalles de un género; 4. Tomar decisiones cuando se requiere escribir de manera innovadora (Bazerman, 2012a, p. 125). Paralelamente, para los docentes, el trabajo con los géneros permite comprender qué están aprendiendo los estudiantes al insertarse, a través de la escritura, en el mundo disciplinar (Freedman y Medway, 2005).

\section{Aportes y dificultades para la didáctica de la lengua}

Queda en evidencia entonces que, tanto para la LSF en Australia, como para la Sociorretórica en Estados Unidos, existe un interés marcado por el reconocimiento explícito de la supremacía de los aspectos socioculturales y el rol del contexto en la comprensión y producción de los géneros. El estudio del significado profundo de los enunciados se encuentra determinado por su inclusión dinámica en la realidad donde son usados. Esto puede tener una ilustración clara en el ejemplo de Mc Cabe (1999, p. 13) desde la LSF, que se asemeja a la situación con la cual Bazerman (2012a, p. 122) ejemplifica la aparición del género. En ambos, se presenta una situación que se soporta y es soportada por el texto escrito, sintetizadas en la siguiente tabla: 
Tabla 1. Ejemplos de producción del género como hecho social

\begin{tabular}{|l|l|}
\hline \multicolumn{1}{|c|}{ LSF (McCabe) } & \multicolumn{1}{|c|}{ Sociorretórica (Bazerman) } \\
\hline Reporte médico. & $\begin{array}{l}\text { Estatutos para la creación de un curso intensivo de } \\
\text { escritura. }\end{array}$ \\
\hline $\begin{array}{l}\text { Etapas para admitir a los pacientes en una sala } \\
\text { de emergencia: chequear signos vitales, tomar } \\
\text { muestras de sangre y orina, entrevistarse con el } \\
\text { doctor. }\end{array}$ & $\begin{array}{l}\text { Criterios que debe cumplir el curso para ser } \\
\text { considerado como intensivo, por ejemplo, un } \\
\text { número mínimo de tareas de escritura. }\end{array}$ \\
\hline $\begin{array}{l}\text { El propósito de estas acciones es determinar si } \\
\text { deben tomarse otras medidas. }\end{array}$ & $\begin{array}{l}\text { El propósito es dejar constancia de los requisitos } \\
\text { para cursar y aprobar el curso. }\end{array}$ \\
\hline $\begin{array}{l}\text { El doctor escribe un reporte para ser leído por otro } \\
\text { doctor. }\end{array}$ & $\begin{array}{l}\text { Los requisitos son puestos por escrito en } \\
\text { documentos administrativos que serán leídos por } \\
\text { estudiantes y maestros. }\end{array}$ \\
\hline
\end{tabular}

Como se ve, los textos se enmarcan en una serie de circunstancias reales y se orientan a cumplir objetivos dentro de esas situaciones. En sentido inverso, los textos también determinan y producen prácticas sociales. En los ejemplos, se evidencia un desapego del uso normativo del lenguaje para prestar atención especial a las acciones sociales que se desarrollan en un circuito permanente de acciones y textos; además, es claro el énfasis de las dos tradiciones en los propósitos que se alcanzan al interior del evento comunicativo.

Sin embargo, las aplicaciones de esta perspectiva social en la investigación y la pedagogía, parecen tener énfasis distintos. En la LSF, de donde se deriva la Escuela de Sidney, aparece un eclecticismo desde el cual se estudia el lenguaje en sus tres dimensiones: ideacional, interpersonal y textual, pero desde las elaboraciones ofrecidas por el mismo texto. Por su parte, la Sociorretórica, que apoya el movimiento WAC, se preocupa por desempacar las relaciones complejas que existen entre el texto y el contexto, atendiendo a los aspectos de la realidad que suceden alrededor de las construcciones linguísticas.

El concepto de género que se propone desde ambos enfoques apunta a objetivos dispares que pueden llegar a complementarse. Para la LSF, se trata de un "proceso social, orientado a objetivos y realizado en etapas" (Martin y Rose, 2012, p. 54); la Sociorretórica lo refiere como una "acción retóricamente tipificada, basada en una situación retóricamente recurrente” (Miller, 1984, p. 27). Así, el análisis del género desde la perspectiva sistémica se "realiza" en el texto mismo, mientras que desde el enfoque sociorretórico se "basa" en la situación exterior. El primer mecanismo permite centrar la interpretación en el texto concreto como objeto de análisis, pero puede dejar en manos de los conocimientos previos del lector la posibilidad de involucrar asertivamente el contexto. Por su parte, la conceptualización de la sociorretórica, presenta una explicación más detallada de la 
situación, aunque no establece pautas claras y concretas de análisis textual. Ambos métodos pueden dar como resultado una descripción general de los textos, pero ambas también pueden dejar al margen aspectos relevantes para la interpretación profunda.

Para la LSF como proceso y para la Sociorretórica como hecho, el género se encuentra vinculado directamente con el contexto social, sujeto a variaciones de tiempo, espacio, actores, intenciones, etc. que lo hacen difícilmente estable y, por tanto, clasificable. Sin embargo, para ser estudiado, y sobre todo, para ser incluido en una didáctica del lenguaje escrito, es indispensable generar un cierto margen de taxonomía que facilite su aprehensión por parte de lectores y escritores nóveles. Para ello, la LSF se interesa por especificar los aspectos constitutivos del texto en función del propósito comunicativo, lo que permite manejar el texto con propiedad, pues se conocen los recursos y las formas lingüísticas que matizan el significado profundo del texto. Por su parte, la Sociorretórica se enfoca en determinar los rasgos característicos de las acciones que dan origen a géneros determinados, esto facilita la reflexión acerca de las demandas de la situación en la que se inscribe el texto. Cabe resaltar además, que el género actúa como nexo entre las acciones individuales y el contexto social definido, por lo que se trata de un fenómeno dinámico en el cual las acciones de los individuos construyen y son construidas de manera recíproca por el contexto de situación, el contexto de cultura y el contexto del género (Devitt, 2004); razón por la cual, el estudio del género no puede realizarse en una sola vía: del texto hacia la realidad o de la realidad hacia el texto, sino que se trata de un mecanismo de interacción recíproco.

De otro lado, es claro que la concepción de género en cada corriente afecta directamente el enfoque pedagógico. Así, la LSF ha logrado puntualizar aspectos muy concretos de la dinámica de aula en la cual el maestro guía detalladamente el proceso de lectura y escritura, enfatizando en los componentes del género: el contexto de cultura y el contexto de situación o registro. Esta estructura pedagógica detallada, brinda un sustento claro tanto al docente como al estudiante que, de un lado, facilita la transferencia de los aprendizajes linguísticos hacia la lectura y escritura de distintos géneros discursivos y, por otro, permite el desarrollo de la autonomía. Por su parte, la Sociorretórica, más enfocada en la dinámica histórica y social del texto escrito no se ocupa puntualmente de los aspectos metodológicos a partir de los cuales generar estrategias para el fomento de la escritura; más bien, postula una serie de características y condiciones de los discursos especializados y sus contextos de producción, que son tenidos en cuenta por los investigadores a la hora de ensayar dinámicas de aula.

Con todo, ambos enfoques abren la posibilidad de leer y escribir para aprender los conceptos de las disciplinas y los entornos comunicacionales que les son propios. 
Derivado de estos conocimientos, contribuyen con una posición liberadora de la sociedad en donde los estudiantes, como sujetos políticos, posean los conocimientos suficientes de su entorno y de su carrera que les permitan tomar posiciones críticas frente a la realidad y actuar en consecuencia. Ambos enfoques luchan por la democratización del conocimiento, una democratización que solo es posible en la medida en que se cuente con el acceso a la información y a la posibilidad de participar en la vida pública.

\section{Conclusiones}

Los campos de estudio de las distintas ciencias ofrecidos como programas profesionales en las universidades, constituyen un espectro bastante amplio de conocimiento, que a su vez está soportado con una gran multiplicidad de textos. Si a esto se suma, tanto la variedad de subdisciplinas de cada ciencia, como los enfoques particulares de cada universidad e incluso de cada maestro, se obtiene un corpus infinito de documentos susceptibles de convertirse en material para ser estudiado durante la formación profesional. Este vasto panorama, genera la urgencia de adelantar procesos de investigación orientados al estudio sistemático de los textos usados para aprender en las universidades. Y es allí donde, tanto la LSF como la Sociorretórica, pueden convertirse en el sustento teórico de las iniciativas en las universidades.

Para un lingüista, no especializado en ninguna otra disciplina, el acceso a estos materiales se torna denso, y más aún el diseño de estrategias metodológicas que desarrollen en los alumnos capacidades para aprender a leer y escribir en sus campos del saber específicos. En este sentido, la LSF permite estudiar los textos desde sus estructuras formales en relación con los contextos de situación; mientras que la Sociorretórica, facilita el estudio de la dinámica académica en relación con el texto escrito. Al fusionar ambos enfoques, se hace posible enfatizar en el estudio del lenguaje desde la LSF y permitir la apertura al análisis de la dinámica social en la que se produce, desde la Sociorretórica; la comprensión del género como proceso en etapas, pero también como hecho perteneciente a una situación tipificada; la rigidez del modelo pedagógico de la sistémica con la posibilidad de trabajar según la dinámica misma del contexto (el contexto del campo de conocimiento y de la asignatura donde se utiliza determinado texto). Y, especialmente, el énfasis marcado hacia la comprensión del lenguaje al interior de la esfera social como mecanismo de acción, pero también como constituyente de la acción. 


\section{Referencias bibliográficas}

Austin, J. (1962). How to do things with words. Cambridge: Harvard university press.

Bazerman, C. (1988). Shaping Written Knowledge. Wisconsin: The University of Wisconsin Press.

Bazerman, C. (2012a). Géneros textuales, tipificación y actividad. México: Universidad Autónoma de Puebla.

Bazerman, C. (2012b). Genre as social action. En: J. Gee y M. Handford. The Routledge Handbook of Discourse Analysis. New York: Routledge.

Bazerman, C., Little, J., Bethel, L., Chavkin, T., Fouquette, D., y Garufis, J. (2005). Reference guide to Writing across the curriculum. Indiana: Parlor Press.

Bazerman, C., Dean, C., Early, J., Lunsford, K., Null, S., Rogers, P., y Stansell, A. (2012). International advances in writing research. Cultures, places, measures. Colorado: The WAC Clearinghouse.

Bazerman, C., y Miller, C. (2011). Géneros textuales. Disponible en: http://www.nigufpe.com.br/ batepapoacademico/bate-papo-volume1-quest4-espanhol.html

Bernstein, B. (2000). Pedagogy, Symbolic Control and Identity: Theory, Research, Critique. London: Taylor and Francis.

Bruffee, K. (1986). Social Construction, Language, and the Authority of Knowledge: A Bibliographical Essay. College English, 48(8), 773-790.

Carranza, I. (2012). Los géneros en la vida social: La perspectiva fundada en las prácticas sociales. En: M. Shiro, P. Charaudeau y L. Granato (eds.). Los géneros discursivos desde múltiples perspectivas, teorías y análisis. España: Vervuert.

Devitt, A. (2004). Writing genres. Carbondale: Southern Illinois University Press.

Durkheim, E. (1986). Las reglas del método sociológico. México: Fondo de Cultura Económica.

Ghio, E., y Fernández, D. (2008). Lingüística sistémico funcional. Aplicaciones a la lengua española. Santa Fe: Universidad Nacional del Litoral.

Freedman, A., y Medway, P. (2005). Genre and the new rhetoric. London: Taylor and Francis.

Halliday, M. (1982). El Lenguaje como Semiótica Social. La Interpretación Social del Lenguaje y del Significado. México: Fondo de Cultura Económica. 
Halliday, M. (1989). Functions of language. En M. Halliday y R. Hasan (eds.). Language, context, and text: Aspects of language in a social-semiotic perspective. Oxford: Oxford University Press. 15-28.

Halliday M., y Hasan, R. (1985). Language, Context, Text: Aspects of Language in a Social Semiotic Perspective. Hong Kong: Oxford University Press.

Malinowski, B. (1923). The problema of meaning in primitive languages. En: C. Ogden e I. Richards (eds.). (1946). The meaning of meaning. London: Kegan Paul. 296-336.

Martin, J. R., y Rose, D. (2008). Genre relations. Mapping culture. Australia: Equinox.

Martin, J. R., y Rose, D. (2012). Learning to write, Reading to learn. Australia: Equinox.

McCabe, A. (1999). Theme and thematic patterns in spanish and English history texts. Disponible en: http://www.isfla.org/Systemics/Print/Theses/McCabephd.pdf

Miller, C. (1984). Genre as social action. En: A. Freedman y P. Medway. (2005). Genre and the new rhetoric. London: Taylor and Francis.

Moyano, E. (2014). Reflexiones en torno al desarrollo de habilidades de lectura y escritura en la universidad. Presentación en el curso de Eficacia Comunicativa. Universidad del Norte.

Navarro, F. (coord.).(2014). Manual de escritura para carreras de bumanidades. Buenos Aires. Facultad de Filosofía y Letras Universidad de Buenos Aires.

Rojas, I. (2015). Leer y escribir en las disciplinas. Estado del arte en las universidades colombianas. En prensa.

Rorty, R. (1989). Solidarity or Objectivity. En: M. Krausz. Relativism, interpretation and confrontation. Notre Dame: University of Notre Dame Press.

Russell, D., Lea, M., Parker, J., Street, B., y Donahue, T. (2009). Exploring Notions of Genre in "Academic Literacies" and "Writing Across the Curriculum": Approaches Across Countries and Contexts. En: C. Bazerman, A. Bonini y D. Figueiredo. Genre in a changing world. Colorado: Parlor Press.

Searle, J. (1969). Speech Acts. Cambridge: University Press.

Schütz, A. [1932] (1993). La construcción significativa del mundo social. Introducción a la sociología comprensiva. Barcelona: Paidós.

Vygotsky, L. (1962). Thought and Language. Cambridge: MIT Press.

Vygotsky, L. (1978). Mind in Society: The Development of Higher Psychological Processes. Cambridge: Harvard University Press. 